\title{
L'expérience : une approche conceptuelle au service du tourisme
}

Experience: a conceptual approach in the service of the tourism

Solène Bargain et Sandra Camus

\section{CpenEdition}

\section{Journals}

Édition électronique

URL : http://journals.openedition.org/tourisme/1387

DOI : $10.4000 /$ tourisme. 1387

ISSN : 2492-7503

Éditeur

Éditions touristiques européennes

Référence électronique

Solène Bargain et Sandra Camus, «L'expérience : une approche conceptuelle au service du tourisme », Mondes du Tourisme [En ligne], 13 | 2017, mis en ligne le 30 décembre 2017, consulté le 20 mars 2020. URL : http://journals.openedition.org/tourisme/1387 ; DOI : https://doi.org/10.4000/tourisme.1387

Ce document a été généré automatiquement le 20 mars 2020.

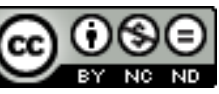

Mondes du tourisme est mis à disposition selon les termes de la licence Creative Commons Attribution - Pas d'Utilisation Commerciale - Pas de Modification 4.0 International. 


\title{
L'expérience : une approche conceptuelle au service du tourisme
}

Experience: a conceptual approach in the service of the tourism

\author{
Solène Bargain et Sandra Camus
}

\section{Introduction}

1 Présenter l'expérience comme une catégorie d'offre à part entière est, depuis le début des années 2000, moins un simple effet de mode qu'un constat communément admis.

2 Les définitions usuelles $d u$ Larousse présentent l'expérience dans une logique interdisciplinaire, l'expérience (du latin experiantia, faire l'essai) étant tour à tour décrite comme la "pratique de quelque chose, de quelqu'un, épreuve de quelques choses dont découlent un savoir, une connaissance, une habitude en vue de tirer une connaissance de cette pratique "; comme "le fait de faire quelque chose, de vivre un évènement considéré du point de vue de son aspect formateur " pour faire écho au plan émotionnel ; ou encore comme " l'action d'essayer quelque chose, de mettre à l'essai un système, une doctrine dans une logique d'expérimentation" sur le plan scientifique. La notion d'expérience peut être ainsi communément appréhendée comme le fait d'effectuer une action, motivé en cela par des objectifs pratiques et émotionnels. Retirer une forme d'apprentissage réexploitable tout en vibrant pendant cette acquisition de compétences.

3 L'expérience est un concept qui fait partie de l'individu au sens propre : celui-ci, au travers de ses actes de la vie quotidienne ou du hors-quotidien, peut être amené à en retirer un apprentissage, résultat d'un ressenti vécu.

4 Dans l'ensemble des disciplines sociales et humaines, la notion d'expérience fait l'objet d'un traitement conceptuel dont les limites de définition sont en constante évolution.

Dans le champ de la littérature, l'expérience est appréhendée au travers du prisme émotionnel par les auteurs dès les années 1800 , avec notamment les écrits des auteurs issus du courant des Romantiques qui, à travers leurs récits de voyage, nous livrent les turpitudes et les émois passionnés des protagonistes. 
6 En anthropologie, la notion d'expérience a été abordée dans les écrits de Boorstin (1964) ; MacCannell (1973) ; Jafari $(1981 ; 1988)$; Turner $(1969$; 1982) et de Graburn $(1976 ; 1983$; 1989) ; ce dernier, tout comme Boorstin, questionnant l'authenticité des expériences dans le tourisme.

7 En marketing, la définition de l'expérience fait l'objet d'une réflexion conceptuelle depuis le milieu des années 1980. Elle prend racine dans les courants de recherche relatifs à l'économie et la psychologie sociale notamment. Le concept d'expérience de consommation a commencé à prendre une place prépondérante en marketing grâce à l'article fondateur d'Holbrook et d'Hirschman (1982). Cette vision expérientielle de la consommation permet de proposer de nouvelles catégories de leviers stratégiques et opérationnels pour les acteurs économiques et institutionnels. En effet, les chercheurs en marketing présentent l'expérience comme un nouvel outil de différenciation venant enrichir la notion de positionnement, présentée de manière traditionnelle comme une démarche essentiellement cognitive (Mencarelli, 2005).

Le présent article a pour objectif, dans un premier temps, de dresser un panorama du courant de recherche de l'expérience dans une logique multidisciplinaire, le concept étant abordé dans de nombreuses disciplines en sciences humaines et sociales. Dans une seconde partie, il s'agira d'étudier en quoi les contributions théoriques de l'ensemble de ces disciplines constituent des repères à opérationnaliser pour les institutionnels comme pour les autres prestataires du tourisme, désireux de positionner leurs réflexions managériales dans le cadre de l'expérience.

\section{Expérience et tourisme : une approche multidisciplinaire en sciences humaines et sociales}

Dans le domaine de la psychologie cognitive et de l'évaluation formative, l'adjectif expérientiel apparait en 1969 avec la citation extraite de l'ouvrage de R.D Laing $L a$ politique de l'expérience qui fait état " des nombreuses variantes pratiques de la psychothérapie longue ou brève, intensive, expérientielle, dirigée ou non..." (Laing, 1969, p. 38). L'expérience est ainsi présentée comme un "mode d'acquisitions de connaissances fondé sur un contact direct avec des réalités et des phénomènes " (Lochard, 2007, p. 82). Dans le contexte des années 1970, l'auteur souligne que la "formation expérientielle » s'inscrit désormais dans un mouvement plus vaste d'idées, autour d'un socle de valeurs reposant conjointement sur "la dénonciation de la société de consommation, de l'aliénation de l'individu mis au service exclusif de la consommation, des richesses dont ils sont porteurs [...] dans une affirmation de la dignité de tous les hommes et des richesses dont ils sont porteurs " (Ibidem). L'expérience personnelle, au travers des apports qu'elle délivre, s'inscrit dans une dynamique de remises en cause, d'inventivité, de foi dans les ressources personnelles. Dans "l'apprentissage expérientiel, l'acte d'apprendre se déroule dans un contexte donné où apprendre nécessite une réflexion sur soi et sur son rapport avec ce contexte " (Ibid.) : l'auteur souligne le caractère innovant de l'expérience, qui propose une mise en question et en recul d'un système au travers de sa perception, en vue d'en retirer des apprentissages.

Les auteurs de philosophie présentent, quant à eux, l'expérience en suivant une approche ambivalente : à la fois point de contact avec la réalité matérielle (nécessaire, semble-t-il, à la constitution d'un savoir) et variable incertaine ou encore fluctuante. 
Aussi, un distinguo s'effectue entre les différentes manières d'approcher l'expérience : expérience immédiate, perception liée à l'expérience, sensation, expérimentation, expérience construite par l'homme et expérience vécue... Autant de formes disparates qui amènent les chercheurs en philosophie à se poser la question de l'enseignement que l'individu peut extraire de l'expérience compte tenu de sa variabilité. À titre d'exemple, le caractère immédiat de l'expérience peut être trompeur car ce que l'individu reçoit est de l'ordre de la perception, image déformée de la réalité. Le savoir scientifique serait donc la résultante de la pensée dans une démarche inductive, l'expérience concrète servant de base pour être ensuite interprétée à l'aune de lois universelles. La question de l'expérience divise dès lors les penseurs, entre rationalistes d'une part et empiristes d'autre part. Le rationalisme, représenté par les écrits de sa figure de proue, Emmanuel Kant, reconnait à "l'expérience qu'elle soit une origine et non un fondement» (Cournarie, 2010, p.7); alors que l'empirisme "soumet la pensée à l'expérience comme fondement et critère ultime de toute connaissance » (Ibid).

De fait, cette présentation de l'expérience comme élément ne produisant aucun savoir d'un point de vue philosophique - la connaissance étant générale et résultant de la pensée - est à nuancer. L'expérience apprend bien à l'individu une forme de savoir, qui n'est pas usuellement défini sous forme de lois mais en tant que matières, dans les connaissances qu'il apporte. L'expérience fournit de fait un apprentissage : c'est le lieu de l'existence qui se vit dans la pratique, à l'instar de Sartre qui précise que "l'existence précède l'essence».

Dans un tout autre registre, l'expérience est intimement liée aux émotions, en particulier lorsque le sujet est le tourisme. Bien que les chercheurs s'accordent à penser que le tourisme expérientiel constitue en soi un "abus de langage ", une "tautologie " (Petr, 2014) en ce sens que le tourisme est, par nature, le fait d'une expérience constitutive d'un déplacement touristique, la notion d'expérience, dans son approche émotionnelle et formatrice, est antérieure à la définition institutionnelle du tourisme.

En effet, la pratique du voyage, bien antérieure à la pratique du tourisme en tant que telle ${ }^{1}$, existe depuis l'époque romaine, avec les séjours dans les stations thermales agrémentés d'excursions dans les alentours (Bertho-Lavenir, 1999). Au XVIII siècle, les aristocrates considèrent quant à eux la pratique du voyage comme un périple à valeur initiatique à travers toute l'Europe, en vue de découvrir ses beautés naturelles et patrimoniales. Dénommé le "Grand Tour ", ce périple offre aux voyageurs la possibilité de parfaire leur éducation artistique et culturelle et par là-même de faire vibrer leur âme " romantique ".

14 Aux siècles suivants, l'art du voyage fait l'objet d'une retranscription littéraire à travers les écrits de Madame de Sévigné ou encore, avant elle, les récits que Montaigne écrivit au gré de ses voyages en Italie. Stendhal, Victor Hugo, Alexandre Dumas puis Flaubert sacrifient à la tradition en rédigeant des textes « dont le récit suit fidèlement la progression géographique et temporelle du narrateur" (Bertho-Lavenir, 1999, p. 44). Si la rédaction reproduit fidèlement et sans surprises les indications concernant les paysages rencontrés, il n'en reste pas moins que le récit dilue progressivement toutes formes d'indications pragmatiques pour céder la place aux critères purement esthétiques et aux ressentis passionnés des narrateurs lors de leurs pérégrinations. Les sentiments exaltés, l'évocation des palettes émotionnelles éprouvées à chaque destination permettent en cela de présenter le voyage dans ce qu'il a d'essentiel pour l'individu: une exaltation des sens, des émotions, intimes témoins des ressentis. Flaubert, dans son 
ouvrage Voyage en Orient - qui retrace son périple en Égypte, Grèce, Constantinople... avec Maxime Du Camp - nous livre ainsi les émotions d'un narrateur tour à tour empreintes d'une profonde mélancolie - le mal du siècle - :

«O Nil! Ma tristesse est débordante comme tes eaux. Et personne non plus ne saurait dire d'où elle vient, c'est au milieu de mon été que l'inondation est accourue [...] ( (Flaubert, carnet 5)

et d'une profonde exaltation à la vue de paysages, à l'instar de l'arrivée à Thèbes :

"C'est alors que, jouissant de toutes ces choses, au moment où je regardais trois plis de vagues qui se courbaient derrière nous sous le vent, j'ai senti monter du fond de moi un sentiment de bonheur solennel qui allait à la rencontre de ce spectacle.» (Flaubert, carnet 5)

L'émergence du chemin de fer (21 février 1804), d'une part, puis des congés payés en 1936 en France, d'autre part, permettent un changement de perspective quant à la pratique du voyage. Le tourisme dit «de masse » s'ouvre aux classes moyennes. Ces nouveaux touristes découvrent à leur tour les promesses que semble délivrer le déplacement touristique : celui d'un espace en dehors du quotidien, au travers duquel la stimulation des sens, l'imagination, sont autant d'éléments favorables à une expérience riche et mémorable.

Pour les chercheurs en tourisme, la question de l'expérience est souvent appréhendée par le prisme de l'authenticité (authenticité de l'objet consommé ou du vécu), les touristes étant à la recherche d'une expérience transcendante, porteuse de ressentis émotionnels et source d'apprentissages.

L'anthropologie se positionne sur l'étude de l'homme en relation avec les populations, les origines, les classifications et les relations entre ethnies, sur son comportement physique, ses relations sociales et environnementales ainsi que dans son rapport à la culture (Jafari et Ritchie, 1981).

19 Certains chercheurs de cette discipline estiment que le tourisme est une activité superficielle (ou perçue comme telle), en marge et triviale par rapport aux problématiques de la société moderne (Boorstin, 1964). Il n'en reste pas moins que la question de la recherche d'expérience pour le touriste fait l'objet de réflexions par une poignée de chercheurs, parmi lesquels on retrouve Boorstin, ainsi que MacCannell. En examinant le tourisme de masse, Boorstin (1964) associe les expériences vécues à des « pseudo-évènements ", du fait de la recherche par l'individu touriste de mises en scènes de manifestations, d'expériences artificielles, de satisfaction par le biais de produits banalisés, de cultures et d'images (Shen, 2011, citant Boorstin, 1964). L'auteur suggère en ce sens que "les touristes sont incapables d'avoir des expériences authentiques" (Ibid, p. 68). De même, MacCannell (1973) corrobore cette assertion en proposant le terme « d'authenticité mise en scène ", de même que Cohen $(1979$; 1988) pour qui les visités, lorsqu'ils se comportent autrement qu'avec leurs semblables, sont censés faire " usage d'une authenticité mise en scène " (Michaud, 2001, p. 19). En s'inspirant de notions telles que le "devant de la scène » et "dans les espaces privés ${ }^{2}$ » - introduites par Goffman (1959) -, MacCannell justifie l'idée selon laquelle «le devant de la scène » (composé du personnel d'accueil et des managers en interaction avec les clients) pourrait être apparenté à une "fausse réalité ", dédiée au spectacle et à la performance en elle-même. À l'inverse, les coulisses seraient l'endroit de la "réalité réelle " pour les acteurs, chargés du spectacle, pour reprendre la métaphore théâtralisée (Goffman, 1959; MacCannel, 1973, p. 589). L'auteur conclut ainsi en démontrant que les touristes, frustrés par leur 
vie routinière en manque d'authenticité, sont néanmoins motivés par la question de l'authenticité, de la spontanéité au sein de leurs expériences touristiques, notamment au travers de leurs relations avec les autochtones. Boorstin (1964), en amont, avait évoqué la question de l'isolement ressenti par les touristes, éloignés à la fois de leur environnement d'accueil et de la population locale en restant dans leurs hôtels et vivant de ce fait des expériences inauthentiques. À l'instar des «anciens pilgrims, les touristes sont à la recherche d'expériences authentiques et d'endroits sacrés transportés dans un hors-temps et un hors-lieu quotidien» (Shen, 2011, p. 69). Graburn (1983) explicite à cet égard l'idée selon laquelle le tourisme devrait être appréhendé au travers de la manifestation par l'individu du besoin de jouer et de se recréer dans une logique de donner du sens, quelle que soit l'activité proposée ainsi que l'origine sociale ou culturelle des acteurs. Cet auteur (1989) caractérise le tourisme comme une sorte de processus de rituels qui reflètent les valeurs profondes de la société, comme la santé, la liberté, la nature, l'amélioration de soi. Les vacances, selon lui, peuvent être interprétées comme les équivalents modernes, et dénués de leur caractère religieux, des festivals et autres pèlerinages dans des sociétés plus traditionnelles et religieuses. En s'inspirant de Durkheim, Graburn analyse "la fonction rituelle du tourisme dans la société, plus spécifiquement dans la construction et le maintien d'une conscience collective" (Stronza, 2001, p. 6). Au travers de symboles et de souvenirs, les touristes sont capables de renforcer leur connexion à eux-mêmes et aux membres de la société. L'approche du chemin initiatique rejoint celle développée par Turner et Turner (1978) pour qui le voyage de loisirs est comme un pèlerinage, qui extirpe les individus de leurs structures ordinaires et quotidiennes. Le tourisme offre à cet égard une liberté en dehors du travail et des obligations habituelles, une découverte de rôles sociaux traditionnels et la possibilité en cela de passer du temps dans cet espace hors du quotidien. Le tourisme moderne reflète une approche à contre-courant de la vie quotidienne, une échappée plutôt qu'une quête (Turner, 1969, 1982).

Finalement, le touriste apparait comme une personne porteuse d'un projet existentiel, à la recherche d'une activité de loisirs conciliant à la fois une activité de récréation et de recréation (Équipe MIT $^{3}, 2008$ ) au sens de "reconstitution du corps et de l'esprit » (Knafou, 1997, p. 198). En effet, le touriste s'emploie à transcender la simple approche récréative d'une activité de loisirs par le biais d'un déplacement touristique. Cette démarche constitue en soi un enjeu de reconstitution, après le travail du corps et de l'esprit, et dont l'effort - conscient et inconscient - relève de ce que l'équipe MIT appelle la «recréation» (Équipe MIT, 2008, p. 104). Jafari (1988) souligne cet aspect de va-et-vient entre un quotidien qualifié de destructeur et un hors-quotidien « recréateur " (Équipe MIT, 2008) qui permet en ce sens aux «travailleurs de se re-créer [...] prêts à réintégrer leur place dans le système principal » (Jafari, 1988, p. 74).

21 La Cofremca ${ }^{4}$ (1990) souligne par ailleurs que les touristes français sont également à la recherche d'un «donner sens » à des moments privilégiés que sont les vacances par leur désir commun d'être différents et de ressentir des expériences rares développant par là-même leur singularité. En résulte par conséquent un nouveau rapport à soi, à autrui et à l'espace, conséquence de nouveaux besoins fonctionnels, de désirs immatériels hétérogènes (Amirou, 2000) et de besoin de sécurisation à travers les thématiques de l'authenticité, de la simplicité et du terroir (Lebrun, 2005). 
L'approche conceptuelle de l'expérience s'inscrit dans une nouvelle approche du marketing, en pleine évolution : celle d'un marketing qui, de transactionnel, devient expérientiel, après avoir été relationnel au cours de ces dernières décennies. (2007), l'anecdote historique relative aux marchands de Sumer qui pratiquaient, il y a six mille ans déjà, le marketing sensoriel en exploitant notamment les couleurs des produits pour organiser l'étal sur un marché, Filser trace un parallèle avec le marketing contemporain, tout en y apportant des nuances. Alors que les commerçants d'antan pratiquaient une forme de marketing sensoriel, le marketing contemporain se distingue par sa capacité à « identifier les processus physiologiques et psychologiques qui sont à l'œuvre lorsque le chaland est au contact des produits ». (Filser, 2007, p. VIII). À cet égard le marketing est de nos jours présent dans les sphères de toute activité marchande et s'intègre désormais dans celles dites non marchandes (Ibid.). C'est dans ce contexte d'ouverture de son champ d'action que la réflexion autour du marketing prend son sens et met à jour une évolution de ses approches conceptuelles.

24 La recherche en marketing a en effet développé l'approche transactionnelle jusqu'à la fin des années 1970. La dimension cognitive a dominé la recherche en comportement du consommateur. Le marketing est ainsi présenté

« comme un processus social dans lequel des systèmes distributifs évoluent pour rencontrer les besoins sociaux et où les participants à ce processus créent des transactions - sous des conditions de contraintes économiques et socio-techniques, résultant de différentes formes d'échanges appréhendés comme différents modes de transferts de biens et de services exécutés en contrepartie et en équivalence les uns des autres. » (Flambart-Ruaud, 1997, p. 8)

5 Le consommateur est en quête d'un produit qui réponde à ses attentes, en fonction de ses besoins et de son budget, sur la base du meilleur rapport qualité-prix. Il est ainsi perçu comme " un individu qui pense de façon logique, suit un processus réfléchi et raisonné en vue de résoudre des problèmes pour aboutir à des solutions " (Filser, 2007, p. VIII). La prise de décision - l'acte d'achat - est expliquée par des modèles de hiérarchie des effets, dans lesquels le consommateur évalue les caractéristiques du produit, celles d'ordre fonctionnel notamment, les compare de manière rationnelle (Ibid.) en vue de prendre une décision suivant une corrélation entre ses besoins exprimés et la manière dont le produit satisfait ces besoins.

6 En 1994, Grönross oppose le «transaction marketing " au « relationship marketing » et relaie l'idée selon laquelle le marketing relationnel serait différent du marketing transactionnel, jusqu'alors utilisé. Or Marion (2001) réfute cette affirmation en arguant d'une pseudo dichotomie entre ces deux approches. Selon lui, "l'opposé de transaction n'est pas relation en cela que toute transaction est enchâssée au sein de relations qui, en tant que phénomènes, font partie de tout échange marchand » (Ibid, p. 12).

Peu à peu, cette appréciation du consommateur a évolué pour s'intéresser au sens donné à l'acte de consommation, au cours duquel la dimension affective et émotionnelle intervient dans le processus de prise de décision, en plus de la dimension cognitive. Les travaux d'Holbrook et d'Hirschman (1982) - dont l'article publié à la même date est considéré comme l'une des contributions fondatrices du courant de l'expérience de consommation - constituent un repère chronologique commun relatif à l'introduction de la réflexion sur l'approche expérientielle. Le consommateur est en quête de sensations mémorables qui transcendent la simple satisfaction résultant de la 
consommation d'un service fonctionnel : il recherche le frisson, l'évasion, l'imaginaire. Les auteurs définissent l'expérience de consommation "comme un état subjectif de conscience, accompagné d'une variété de significations symboliques, de réponses hédonistes et de critères esthétiques » (Holbrook et Hirschman, 1982, p. 132).

Alors que la recherche en consommation met l'accent sur les bénéfices tangibles de biens conventionnels et autres services, qui remplissent des fonctions utilitaires sur la base de caractéristiques relativement objectives, la perspective expérientielle explore, quant à elle, les significations symboliques de caractéristiques plus subjectives (Ibid.). La recherche traditionnelle en consommation s'est concentrée sur les attributs de produits qui tendent à des descriptions verbales. Mais les analyses conjointes et les modèles multi-attributs ont beaucoup misé sur le design, qui use de stimuli verbaux. Bon nombre de produits projettent d'importants indices non verbaux qui peuvent être vus, entendus, testés, ressentis, sentis en vue d'être appréciés. Ainsi, dans beaucoup de situations de consommation, à l'instar d'un visionnage de film ou d'une dégustation dans un restaurant, différentes chaînes sensorielles opèrent simultanément (Ibid.).

Envisagée comme un vécu individuel, l'expérience de consommation met l'accent sur le vécu personnel en occultant parfois la relation entre l'objet et la situation de consommation (à l'origine des recherches en tout cas), éléments introduits par Punj et Stewart (1983) dans leur paradigme «Personne x Objet x Situation ».

Suite à l'émergence de cette réflexion, les économistes Pine et Gilmore (1998), dans leur article pionnier, identifient une ère économique nouvelle qualifiée d'économie de l'expérience - constituée d'expériences - qui diffère de l'économie des biens et autres services (voir figure 1). Les auteurs développent l'idée d'une approche évolutionniste de l'économie en montrant qu'historiquement les biens manufacturés (goods), qui ont remplacé les matières premières (commodities), sont eux-mêmes remplacés par les services, puis par les expériences (experiences). Ils expliquent que les entreprises doivent mettre en scène l'expérience afin d'obtenir un bénéfice complet, lié à la création de valeur au travers d'expériences pour leurs clients. La définition de l'expérience de consommation selon Pine et Gilmore met à jour une composante essentielle qui est la participation du client, dont le degré d'investissement se situe sur un continuum allant d'une dimension passive de son activité, d'un côté, à une dimension active, de l'autre côté, dans laquelle il a un rôle-clé à jouer. Les clients qui se situent au milieu de ce continuum contribuent, par leur présence, à l'évènement visuel et auditif de l'expérience. Pine et Gilmore, à cet égard, identifient cinq composantes essentielles qui doivent s'agréger autour de l'expérience et qui constituent l'habillage expérientiel: la thématisation, une impression unifiée positive, l'élimination des éléments négatifs, la production de souvenirs que les consommateurs pourront emmener avec eux et la sollicitation des cinq sens. Le caractère de mémorabilité (Batat et Frochot, 2014) constitue un élément essentiel pour la réussite de l'expérience. 
Figure 1. L'économie expérientielle d'un produit ou service - Matrice de Pine et Gilmore (1998)

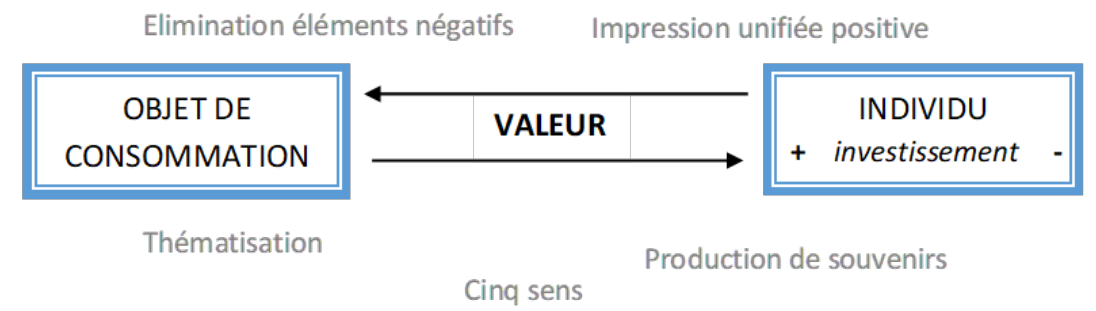

Habillage expérientiel

31 À la suite de cette réflexion, Arnould et al. (2002) indiquent que l'expérience de consommation s'inscrit dans une approche longitudinale où la dimension temporelle est répartie en quatre périodes : la phase d'anticipation qui renvoie aux "actions de recherche d'information, d'optimisations budgétaires, de planifications et d'attentes en termes d'expériences futures » (Batat et Frochot, 2014, p. 6) ; l'expérience d'achat - processus de sélection, de choix du produit et d'interaction avec les autres éléments du lieu de vente (Ibid.); l'expérience vécue en elle-même par le consommateur; et l'expérience de souvenir pour revivre l'expérience antérieure via les photos et autres éléments facteurs de mémorabilité (Ibid.).

32 Carù et Cova (2002), quant à eux, définissent l'expérience de consommation comme un vécu personnel - souvent chargé émotionnellement - fondé sur l'interaction avec les stimuli que sont les produits ou les services rendus disponibles par le système de consommation. Ils soulignent en outre que l'expérience de consommation varie sur un continuum allant d'une expérience principalement orchestrée par l'entreprise (company-driven experience), dans laquelle le consommateur joue un rôle considéré comme passif, à une expérience mémorable et totalement produite par le consommateur à des fins d'immersion (consumer-driven experience). Entre les deux, se trouvent des expériences co-produites par l'entreprise et le consommateur, pour lesquelles l'entreprise fournit les conditions de l'expérience, sous forme de plateforme expérientielle (Carù et Cova, 2007), qui sont susceptibles d'être appréhendées par le consommateur en vue de créer sa propre expérience.

Ainsi, pour ces auteurs, une expérience est produite lorsque l'entreprise utilise intentionnellement des services en tant que scène et des produits en tant qu'accessoires pour entraîner des clients individuels dans la création d'un évènement mémorable. Si les matières premières sont fongibles, les biens tangibles, les services intangibles, les expériences sont quant à elles qualifiées de mémorables. De même, alors que les trois premières formes d'économie - matières premières, biens et services - sont externes à l'acheteur, les expériences sont, elles, intrinsèquement personnelles, existant uniquement dans l'esprit d'un individu qui s'engage à un niveau émotionnel, physique, intellectuel, voire spirituel (Ibid.). À cet égard, la première dimension de l'expérience de consommation est la résultante d'une interaction entre un évènement scénique (à l'instar d'une pièce de théâtre) et l'état d'esprit de l'individu. La seconde dimension est la connexion, ou relation, dite d'environnement, qui relie le client à l'évènement ou à la performance au point de s'immerger.

Pour concevoir une expérience de consommation, les entreprises combinent un ensemble de produits et de services autour d'un thème porteur, en utilisant l'ensemble 
des variables du marketing mix - à savoir: prix, produit, positionnement, communication - et en intégrant notamment la variable " atmosphère des lieux » dans lesquels se déroulent les expériences (Kotler, 1973). La métaphore du spectacle est largement mobilisée en vue de promouvoir l'idée qu'une expérience s'apparente à une mise en scène d'un temps extraordinaire (Pine et Gilmore, 1998, 1999 ; Wolf, 1999).

Selon Arnould et Thompson (2005), l'approche expérientielle appartient au courant de la théorie des cultures de consommation (Consumer Culture Theory), qui transcende l'optique utilitariste jusqu'alors appréhendée dans la recherche en comportement du consommateur au profit de la prise en compte des dimensions idéologiques, socioculturelles, symboliques et expérientielles relatives à la consommation.

$\mathrm{Au}$ fil du temps, le consommateur est ainsi de plus en plus perçu comme un être émotionnel à la recherche d'expériences sensibles (Maffesoli, 1990), pour qui la consommation est appréhendée comme un processus de production d'une expérience plus ou moins gratifiante (Filser, 2002) par laquelle il se trouve transformé (Firat et al., 1995). Néanmoins, les expériences sont considérées comme plus ou moins gratifiantes en ce sens que chaque acte de consommation n'engendre pas le même caractère mémorable expérientiel. De même, la transformation n'est pas aussi totale pour chaque individu à chaque expérience vécue. Aussi, Filser (2002) propose de situer ces expériences sur un continuum allant de l'expérience ordinaire à l'expérience extraordinaire, en passant par des produits à habillage expérientiel (décor, intrigue, action), en fonction de l'intensité de l'expérience identifiée.

Suite à ces travaux, Filser (2002) définit l'expérience de consommation comme la série de conséquences positives ou négatives retirées de l'usage d'un bien ou d'un service. Le consommateur est présenté comme le producteur de sa propre expérience subjective, caractérisée par l'intensité des réponses émotionnelles qu'elle provoque chez lui. S'appuyant sur les travaux d'Holbrook (2000) qui identifient quatre composantes de la production d'expérience qualifiant selon lui l'offre touristique - l'expérience (évasion de la réalité, émotions, plaisir), le divertissement (esthétique, excitation, ravissement), l'exhibitionnisme (porter aux nues, exprimer, découvrir) et l'évangélisme (éduquer, donner l'exemple, garantir) -, Filser (2002) relève deux composantes constitutives de tout acte de consommation d'un produit: une composante expérientielle et une composante non expérientielle.

Il prend pour exemple les restaurants ouverts par des marques dans le secteur de l'agro-alimentaire, qui illustrent les quatre composantes de la production d'expérience d'Holbrook (Filser, 2002, p. 17) :

« Si la dimension expérience est associée à la consommation du repas, source d'évasion de la vie quotidienne, d'émotions et de plaisir, le divertissement n'en reste pas moins une composante intrinsèque de la sortie au restaurant pour lequel le cadre de l'établissement, l'ambiance associée à la foule contribuent à faire du repas au restaurant une sorte de spectacle mis en scène par l'entreprise [...] le restaurant est une forme de spectacle vivant qui se renouvelle sans cesse. Deux repas dans le même restaurant se ressemblent peu. L'exhibitionnisme est une composante moins intuitive avec d'abord une mise en avant du produit [...] à travers sa réalité physique. Mais il y a aussi une exposition du consommateur qui se montre à d'autres individus dans sa relation avec le produit. [...] enfin l'évangélisme est sans doute l'une des composantes majeures du positionnement de ces restaurants. Ils ont une valeur d'exemplarité en ce sens qu'ils montrent comment le produit peut être fabriqué, comment son contenu strictement fonctionnel peut être enrichi par une fonction hédoniste [...]. » 

le marketing dit traditionnel et le marketing expérientiel résultant de l'approche conceptuelle de l'expérience. Les auteurs s'appuient pour ce faire sur les écrits de Schmitt (1999) qui, pour justifier la distinction entre ces deux approches conceptuelles, identifient trois facteurs reposant sur: la nature du produit, le comportement du consommateur et la concurrence sur le marché. Batat et Frochot (2014, p. 3) relayent ces assertions en dénombrant quatre éléments de classification : si, pour le marketing traditionnel, l'accent est mis sur les attributs fonctionnels et les bénéfices produits, pour le marketing expérientiel il repose sur les expériences du consommateur en tant que telles. De même, alors que pour le marketing traditionnel le produit relève d'un assemblage étroit entre la catégorie et la concurrence, pour le marketing expérientiel la consommation relève d'une expérience holistique. En ce sens, le consommateur est appréhendé comme un être irrationnel et émotionnel, alors que dans le marketing traditionnel il est présenté comme un décideur rationnel. Enfin, la méthode d'analyse du marketing expérientiel relève d'une dimension éclectique, tandis que le marketing traditionnel s'appuie sur des outils et des méthodes analytiques, quantitatifs et verbaux.

\section{Le marketing expérientiel, un véritable enjeu managérial}

41 Bhattacharjee et Mogilner (2014) présentent le marketing expérientiel comme l'opportunité de faire de la consommation une série d'immersions extraordinaires pour le consommateur, ce dernier étant engagé dans des processus inoubliables qui lui offrent une expérience.

De même, Hetzel (2002), Lasalle et Britton (2003), Schmitt (2003), Carbone (2004) consacrent de nombreux travaux aux apports managériaux de l'approche expérientielle. La démarche expérientielle peut en effet représenter un avantage concurrentiel commercial (Trinquecoste, 1999), permettre de fidéliser ou de réduire le taux d'attrition des clients et constituer un levier de différenciation (Prahalad et Ramaswamy, 2004; Vargo et Lush, 2008). Lipovetski (2006) présente le marketing expérientiel comme une réponse à des besoins émergents :

« de plus en plus de produits ou de services sont explicitement conçus pour créer de l'expérience vécue [...] on théâtralise les lieux de vente dans le but de créer une ambiance festive [...] dans ce contexte émotionnaliste, la création culturelle présente un immense avantage: celui d'offrir un spectacle renouvelé, de l'étonnement, du dépaysement. "

43 Filser $(2008$, p. 3) relativise cependant la réussite des stratégies expérientielles en indiquant que certaines sont dépourvues d'effet positif. L'auteur nuance l'efficacité des stratégies de création d'expérience menées pour renforcer la position concurrentielle d'un produit ou d'un service, les « résultats opérationnels de ces stratégies étant souvent 
décevants", ce qui conduit les marques et enseignes à abandonner les stratégies expérientielles maximalistes au profit de dispositifs d'offres plus simples et rentables (Kozinets et al., 2002).

Rodererer (2012) souligne cependant que les stratégies expérientielles ont pu avoir des résultats concluants, comme c'est le cas pour la chaîne Starbucks qui est présentée par son fondateur Howard Schultz comme une expérience holistique de dégustation d'un café dans des lieux dédiés propices à la détente et à la rêverie diurne, complétée d'une option de vente à emporter (Ibid.); ou encore comme la municipalité d'Helsinki, désignée comme capitale européenne de la culture en 2002, qui a proposé une multitude d'évènements allant de la construction d'une église de neige à des spectacles sons et lumières (Ibid.). Certains échecs ont néanmoins pu être relevés, comme pour les restaurants Planet Hollywood ou le Hard Rock Cafe, pour lesquels les restaurateurs parlent « d'une expérience de shopping mais échouent à créer un thème qui lie les présentations de merchandising disparates en une expérience de mise en scène ${ }^{5}$ » (Pine et Gilmore, 1998).

Le secteur des grandes enseignes de distribution (Dupuis et al., 2004) et de services a également fait l'objet d'une attention soutenue de la part des chercheurs en marketing, notamment en ce qui concerne la consommation de produits culturels comme le théâtre (Bourgeon et al., 1995), le spectacle sportif (Bourgeon-Renault et al., 2007), la visite d'un musée (Bourgeon-Renault et al., 2009) ou les restaurants du consommateurtouriste (Matson-Barkat, 2012).

Mais le concept d'expérience s'avère utile pour la réflexion marketing des acteurs du tourisme (Petr, 2014), à travers des opérations de «stimulation expérientielle » ou la création d'une offre ayant un "positionnement expérientiel » (Ibid.). De facto, il s'agit pour les acteurs du tourisme d'aborder la problématique de la prestation touristique à travers un prisme nouveau, conciliant deux approches: comprendre l'émotion humaine, développer un vrai sens de l'hospitalité, de la créativité et du spectacle tout en conservant un raisonnement économique et réaliste (Fux, 2014).

Les différentes contributions disciplinaires nous ont permis de dresser un panorama conceptuel de l'expérience. En s'appuyant sur leurs ressentis et leurs vibrations émotionnelles, l'expérience constitue une forme d'apprentissage pour les individus. Les touristes, à travers leurs déplacements touristiques, sont à la recherche de pratiques capables de transcender leur rapport à l'acte de voyager afin de dé-routiniser le quotidien, d'une part, et de se former à de nouvelles manières de percevoir l'environnement spatial et humain qui les entoure, d'autre part. Les touristes sont désireux de ressentir des expériences authentiques afin de bénéficier d'un apprentissage favorable à la recréation de l'être au travers d'un moment de récréation (équipe MIT, 2008).

Dans la seconde partie de l'article, nous proposons différents outils de gestion susceptibles d'être mobilisés par les acteurs institutionnels et les autres prestataires du tourisme désireux de renforcer la portée du concept d'expérience dans leurs activités. 


\section{L'opérationnalisation d'outils de gestion au service de l'expérience touristique}

49 La mise en place d'une démarche expérientielle passe par la prise en compte de différents outils et processus. La participation de l'individu et la mise en scène sont sans doute ceux dont le rôle est le plus fondamental.

\subsection{La place du touriste dans le processus expérientiel}

50 Pine et Gilmore (1998) ont identifié une caractéristique essentielle du concept d'expérience : la participation de l'individu, qui peut être plus ou moins active. Celui-ci peut adopter une attitude passive, se positionner en tant qu'acteur ou encore contribuer par sa seule présence à l'évènement de l'expérience.

51 Cette prise de position du consommateur par rapport à la production de service initie l'approche en termes de "Service-dominant logic » (logique de service dominant, ou SDL) introduite et développée par Vargo et Lusch en 2008. Celle-ci s'oppose à l'approche traditionnelle GDL (Good-dominant logic) : les consommateurs n'achètent plus des biens ou des services pour répondre à des besoins tangibles, mais ils sont à la recherche d'expériences mémorables qui leur font accéder à des espaces d'échange, de création et de partage de valeur avec les entreprises (Batat et Frochot, 2014). De facto, le consommateur est associé aux «dispositions, aux capacités créatives de valeurs [...] il lui revient de fait d'être considéré comme un partenaire compétent » (Ibid., p. 21). Il s'agit ici de souligner la place essentielle de l'individu dans ce processus, qui peut aller jusqu'à relever de la co-création de valeur et d'expérience.

En effet, le comportement de l'individu tend à évoluer. Il apprend plus vite, s'approprie les outils technologiques, se tient informé de manière instantanée. Son rapport aux valeurs et à sa place, au regard de la chaîne de consommation, se modifie. De consommateur lambda, l'individu se transforme en producteur à temps partiel. Ainsi, de Certeau (1990) et de Certeau et al. (1994) font état d'un consommateur «bricolant entre les mailles $d u$ filet ». Cette métaphore est utilisée par les auteurs pour identifier un système représentatif de la société technocratique auquel l'individu ne peut échapper. Il lui appartient par conséquent d'utiliser autant qu'il le peut les "brèves failles qui apparaissent dans le filet» (Ratti, 2002, p. 25). Le consommateur, bien que dominé et n'ayant pas accès au monde de la production, entreprend néanmoins une forme de résistance en s'appropriant les objets à travers l'usage qu'il en fait, qui n'est pas nécessairement celui initialement prévu. L'individu ne se considère plus comme un sujet passif disposant d'objets et soumis à un pouvoir qui le dépasse, il est sujet et acteur. Rifkin (2016) aborde notamment la question des plateformes collaboratives qui inaugurent une perception différente de l'individu : celui-ci est désormais plus disposé à être autonome et indépendant par rapport au système de production dans lequel il était jusqu'à présent immergé, sans possibilité d'y contribuer. Le marketing expérientiel doit donc intégrer cette nouvelle figure du consommateur. Il s'agit de susciter des points de congruence entre, d'une part, ses attentes fonctionnelles et émotionnelles, vecteurs de sens, d'identification et suscitant l'imagination en vue du réenchantement de son quotidien (Ritzer, 2010) et, d'autre part, les schémas marketing proposés. Le consommateur devient, en ce sens, un partenaire essentiel pour l'organisation, afin de participer à la création de la valeur. 
53 Le marketing expérientiel positionne son cadre d'action dans une approche relationnelle, par laquelle l'entreprise souhaite et s'efforce de construire un lien personnalisé et durable avec chaque consommateur.

Dans leur définition de l'expérience de consommation, Carù et Cova (2002) ont établi un continuum du degré de participation de l'individu selon que l'expérience est produite totalement ou partiellement par l'entreprise. (voir encadré 1).

Dès lors, le marketing expérientiel s'inscrit dans une dynamique d'implication du touriste à l'expérience. Celui-ci est positionné au centre de la stratégie entrepreneuriale et considéré comme un acteur à part entière.

\section{Encadré 1. Consumer ou Compagny-driven experience}

Dans la chaîne de restauration « Dans le noir " ${ }^{6}$ implantée dans différentes villes européennes (Paris, Londres, Barcelone, Madrid) et en Russie (Saint-Pétersbourg), l'individu est invité à s'immerger dans l'univers des non-voyants le temps d'un repas. Guidé par un personnel non-voyant, l'individu découvre et appréhende différemment une activité pourtant coutumière, celle d'aller au restaurant. Privé de la vue, l'individu, le temps d'un voyage invisible pour les yeux, va utiliser ses autres sens ; le goût, l'ouie, l'odorat, le toucher pour trouver des repères et un cadre. L'individu est réellement acteur de son expérience. C'est par son désir d'immersion et sa bonne volonté à participer qu'il va pouvoir ressentir, évoluer et apprendre de cette expérience.

À l'inverse, au parc d'attractions du Puy du Fou' (Vendée, France), l'individu est considéré comme un spectateur dans la quasi-totalité de ses actions. Il assiste aux spectacles sur des gradins, n'interagit que peu avec les comédiens-guides, hormis aux points de communication tels que les lieux de restauration, les bornes d'accueil. Son ressenti se fait à travers sa perception cognitive, émotionnelle et multisensorielle des manifestations qui lui sont présentées. C'est au travers de ses déambulations dans les différents lieux reconstitués (« le bourg de l'an 1900 », « la cité médiévale » ou le « fort de l'an mille ») que l'individu va, en mobilisant son imagination, se transporter dans un univers hors-quotidien. Le décor, l'intrigue et l'action sont prévues par l'organisation du Puy du Fou pour permettre au touriste de ressentir sa propre expérience individuelle, librement et à son rythme.

De plus, les cinq facteurs principaux proposés par Pine et Gilmore (1998) sont à prendre en compte dans la dynamique que les entreprises doivent impulser pour offrir un produit expérientiel (Batat et Frochot, 2014): la thématisation de l'offre, l'harmonisation des impressions avec des éléments positifs en vue de laisser chez le consommateur des impressions marquantes, l'évitement d'éléments négatifs susceptibles d'altérer la qualité perçue de l'expérience, le panel de souvenirs (produits et articles), métonymies de l'expérience vécue et l'engagement des cinq sens (l'implication forte de ces derniers étant facteur de renforcement de mémorabilité de l'expérience). Les entreprises ont tout intérêt à suivre ces directives pour renforcer l'habillage expérientiel de leur offre. À cet égard, la chaîne de magasins « Nature et Découvertes » s'est illustrée dans cette pratique de l'expérience (voir encadré 2).

Encadré 2. La matrice de Pine et Gilmore (1998) : le cas de « Nature et Découvertes"

La chaîne de magasins « Nature et Découvertes » a été créée en 1990 par Françoise 
et François Lemarchand « convaincus qu'il est possible de faire du commerce dans le respect de l'homme et de la nature ${ }^{8}$ ». Ils ouvrent le premier magasin « Nature \& Découvertes ", à l'atmosphère accueillante et atypique afin d'inviter les citadins à une reconnexion avec la nature. Dans chaque boutique de la chaîne, la même stratégie expérientielle est mobilisée, même si, au dire de la directrice marketing Françoise Vernet, « François Lemarchand travaille beaucoup à l'intuition » (Rieunier, 2004, p. 80).

Le visuel est stimulé : chaque magasin « Nature et Découvertes » est perçu « comme une caverne d'Alibaba où s'amoncellent des produits originaux et très nombreux » (Ibid., p. 77). L'agencement de différents matériaux naturels, tels que le bois et la pierre, rappellent le message lié à la Nature. La sollicitation des autres sens se traduit notamment par la dégustation offerte de la tisane aux sept plantes, permettant de « créer un accueil agréable et de transmettre les valeurs liées à la convivialité » (Ibid., p. 78). Le toucher, quant à lui, est sollicité à travers la mise à disposition d'articles centrés sur des étagères ou des podiums afin de faciliter leur manipulation. De même, des produits sont laissés à la libre utilisation des consommateurs à l'instar de sièges massants ou chauffants, de CD musicaux en libre-écoute sur des bornes prévues à cet effet, de petites huiles odorantes sous forme d'échantillons. L'odorat est particulièrement sollicité avec la diffusion dans tout le magasin de l'offre sensorielle « Nature et Découvertes », telle que « senteur cèdre ", véritable signature olfactive (Rieunier, 2004). Enfin, des sons rappelant les éléments de la nature (chants d'oiseaux, du vent et de l'eau...) et autres sonorités en lien avec l'univers du magasin (musique traditionnelle apaisante)

accompagnent la déambulation des consommateurs.

L'ensemble de ces éléments, l'expérience vécue de reconnexion à la Nature, dans un espace qui propose une pause dans le quotidien, ainsi que l'interaction avec les vendeurs, dont la présence discrète est néanmoins bienvenue, s'inscrivent dans la création d'une offre expérientielle. En s'appuyant sur les cinq critères développés par Pine et Gilmore (1998), on retrouve en effet :

- la sollicitation des cinq sens ;

- la thématisation de l'offre : le retour à la Nature et à soi, en réaction à une société marquée par l'hyperréactivité, l'hyperconsommation, la perte de repères et d'identité ;

- la création de mix souvenirs avec la possibilité de ramener des petits flyers ou autres éléments de communication sur les produits du magasin ;

- l'évitement des indices négatifs, la nature étant perçue comme hospitalière à partir du moment où l'on est suffisamment préparé en amont (les produits « Nature et Découvertes », livres, accessoires, vêtements, etc., sont autant d'éléments facilitant le retour à la Nature) ;

- l'harmonisation des impressions et des indices positifs : la présence chaleureuse, accueillante et néanmoins discrète des vendeurs, tous sensibilisés à la Nature, constitue un élément susceptible de renforcer l'impression positive que le magasin « Nature et Découvertes » crée chez le consommateur. 


\subsection{Le rôle de la mise en scène dans l'approche expérientielle} 1998, 1999 ; Wolf, 1999). L'exemple de l'Abbaye Royale de Fontevraud illustre très bien la manière dont les supports de la mise en scène (décor, intrigue, action) participent à la création d'un contexte expérientiel (voir encadré 3).

Encadré 3. Les supports de l'expérience de l'Abbaye Royale de Fontevraud L'Abbaye Royale de Fontevraud $^{9}$, cité monastique située près de Saumur (Maine-etLoire), constitue un excellent exemple de mise en scène de l'offre patrimoniale au service de l'expérience. Les touristes sont déjà immergés dans une expérience hors du quotidien grâce à l'environnement architectural, témoignage bâti du XII ${ }^{\mathrm{e}}$ siècle (décor).

Le lieu invite en outre, par sa scénographie épurée, à vivre une expérience hors du temps présent, faite de recueillement et de reconnexion à la nature et à soi (intrigue). Le slogan de la cité « L'émotion est dans l'inattendu » met l'accent sur la promesse d'une vibration émotionnelle imprévisible, ce qui conforte l'envie d'originalité et de dépaysement spatial et cognitif chez le touriste.

Le personnel, dans son interaction mesurée, accueillante et chaleureuse avec le touriste, illustre les valeurs réactualisées d'hospitalité et de générosité, chères à Robert d'Arbrissel, le fondateur de la cité (action). De plus, les membres du personnel assument un rôle qui va bien au-delà de la simple fonction de renseignement : ils sont considérés par les managers comme de véritables guides pour inviter « l'hôte ${ }^{10}$ » de Fontevraud à ressentir sans frustration l'expérience présentée. Ils sont encouragés en cela à transcender une approche fonctionnelle et pragmatique et à créer une connexion émotionnelle avec l'hôte. En s'inspirant du décor environnant, en s'adaptant aux modalités d'interactions du touriste, le personnel s'emploie à instaurer un lien de confiance et à encourager l'immersion de l'hôte par une animation des espaces et des émotions.

Il s'agit donc, pour les organisations, de mobiliser des outils en vue de favoriser et d'intensifier la production d'expérience.

Le développement de scripts de service permet d'effectuer un inventaire des éléments et des ressources, matériels comme humains, en vue de les organiser de manière conjointe et en interaction avec le touriste. «La qualité esthétique, la cohérence visuelle de l'environnement physique» (Fux, 2014, p. 19) sont des conditions dites sine qua non pour renforcer un contexte expérientiel satisfaisant pour ce dernier. Bouder-Pailler et Damak (2004) parlent à cet égard de la dimension visuelle et esthétique du lieu, comme autant de "stimuli qui composent l'environnement physique et qui vont solliciter les sens de l'individu » (Mencarelli et Marteaux, 2005, p. 172) et que l'on peut apparenter au design 
du lieu. Tout lieu concoure ainsi à l'expérience touristique, y compris les espaces dits «intermédiaires" tels que les halls d'accueils. Alors qu'on aurait tendance à les percevoir comme des espaces sans identité ni relation, les halls d'hôtels - définis par Ladwein (2002) comme des non-lieux transitionnels ayant une fonction de rupture entre deux espaces - occupent pourtant une place essentielle. Ce sont autant d'espaces que l'organisation touristique se doit d'appréhender comme des leviers potentiels de création et de vie, à intégrer dans le design d'expérience. La théâtralisation de l'ensemble des lieux participant de l'expérience totale représente donc un enjeu essentiel d'attractivité des touristes (voir encadré 4).

\section{Encadré 4. La mise en scène dans les non-lieux transitionnels}

$\mathrm{Au}$ Quai à Angers (espace de création, de spectacles et de rencontres pour tous les publics), une réflexion sur l'avenir du forum (grand hall d'accueil du Quai) devenait essentielle pour rendre ce lieu de passage (jusqu'alors vide) vivant, animé et ouvert à tous de manière permanente. Selon le directeur Frédéric Bélier-Garcia «le forum présente des volumes immenses et est accessible en entrée libre. Cela permet d'imaginer énormément de choses pour faire du Quai un véritable lieu de $v^{1 e^{11}} »$. Ainsi, l'installation d'une librairie est venue compléter l'offre d'accueil déjà mise en place avec un espace dédié, pourvu d'informations signalétiques et pratiques. De plus, et en référence à cette nouvelle dynamique culturelle d'appropriation des lieux, le spectacle de lancement de saison y est programmé, de nombreux événements y sont organisés (mini-concerts, improvisations, expositions, café-débats...), puis des dispositifs sont mis en place pour susciter les initiatives individuelles et collectives des publics (mise à disposition de canapés, grandes tables, espaces de créations artistiques spontanées...). Cet espace, auparavant appréhendé comme un non-lieu, un couloir de transition entre l'extérieur du bâtiment et les salles de spectacle, devient à son tour un élément participant de l'expérience du lieu. La culture entend être présente et accessible à tous, et l'agencement des lieux lui-même illustre cette volonté.

Ces supports de mise en scène permettent en outre de préserver une large part de spontanéité pour le touriste, afin que celui-ci s'approprie l'espace dans lequel il est et l'histoire dans laquelle il tient le premier rôle.

Par ailleurs, le récit du lieu est animé par le personnel de contact, qui est présenté comme un véritable personnage de l'histoire (Fux, 2014). Bien au-delà de sa fonction professionnelle traditionnelle, le rôle du personnel de contact est un enjeu essentiel de l'animation d'un univers répondant aux attentes du touriste. Facilitateurs, guides accompagnant la production d'expérience (Arnould et al., 2002; Carù et Cova, 2003, 2006) sont autant de termes définissant le personnel de contact. Celui-ci a la capacité d'intensifier les émotions ressenties en transportant le visiteur dans des univers féeriques et enchantés (Pulh et Mencarelli, 2010). En outre, en référence à la métaphore théâtrale introduite par Pine et Gilmore (1999), le personnel de contact doit croire en son texte et être engagé dans la pièce tout en gardant à l'esprit les éléments constituant sa fonction première d'accueil et de renseignements. Un subtil mélange s'effectue entre ces deux approches: répondre aux attentes pragmatiques, prosaïques du touriste (questions concernant l'hébergement, la restauration, les endroits à visiter...) tout en l'invitant à la découverte du contenu émotionnel de l'expérience touristique elle-même (voir encadré 5). 
Encadré 5. Le rôle et la formation du personnel de contact dans la production d'expérience

À Disneyland Paris, la problématique des « cast members » fait l'objet de protocoles et de rites « d'initiation ou [...] de passages » qui visent à ce que le salarié démontre son engagement et sa conviction dans son rapport au groupe Disney. Disney Université garantit une formation autour de la «tradition Disney » afin de perpétuer la mémoire du créateur (Chesneaux, 1997). Disneyland Paris veille à ce que les cast members adoptent des comportements-types, au travers du langage notamment : « Eurodisney est une scène, dont les acteurs accueillant les visiteurs sont sur scène tandis que les acteurs de soutien logistique sont en coulisses. Nous recevons un public [...] composé de visiteurs [...]. Nous portons des costumes [...] nous proposons des aventures [...] » (Ibid., p. 7). Au travers des formations, des uniformes, des protocoles d'action mis en place par l'organisation, le personnel salarié est amené à s'identifier comme un acteur à part entière, participant à la réussite de la mise en scène théâtralisée de l'expérience.

À l'Abbaye Royale de Fontevraud, les membres du personnel de contact sont invités à s'immerger dès leur arrivée dans l'atmosphère du lieu en passant une nuit dans l'hôtel du site patrimonial, en dînant dans le restaurant et en effectuant des visites avec des guides. Il s'agit en effet qu'ils puissent ressentir de manière personnelle l'expérience Fontevraud pour devenir par la suite de vrais ambassadeurs du site. De plus, les managers les encouragent à s'approprier les lieux, en connaissant la chronologie du lieu ou en identifiant les différentes activités développées sur place. Mais, au-delà d'une simple appropriation pratique, les membres du personnel de contact sont également encouragés à distiller dans leur logorrhée des anecdotes sur le site et sur les alentours, à créer un lien plus spontané et chaleureux avec l'hôte. Au restaurant du site, par exemple, le chef de rang $^{12}$ et le maître d'hôtel théâtralisent la présentation des plats, lors de la prise de commande, en émaillant ceux-ci de références liées au site et en invitant l'hôte à imaginer l'histoire du lieu dissimulée derrière les mets culinaires. Il s'agit à cet effet de trouver un subtil équilibre entre la délivrance d'informations techniques dans un temps restreint (ne pas passer trop de temps à chaque tablée au risque de ralentir le service), l'atmosphère historique et émotionnelle, la patience, l'attention des hôtes. Ainsi, un petit entremets composé de morceaux de radis assemblés pour dessiner la tenue d'une moniale devient un challenge pour le chef de rang chargé de sa présentation : il lui faut évoquer les différents produits alimentaires, faire deviner l'allusion, rebondir sur des anecdotes, interagir avec l'hôte. L'exercice, peu aisé en soi, demande des talents d'improvisation, d'adaptation, de théâtre : le personnel ne récite pas un texte mais l'interprète en se servant du contexte spatial, émotionnel et culinaire, ainsi que de la réaction de ses hôtes.

Cette présentation d'outils et de conditions susceptibles de contribuer à la réussite du développement d'un contexte expérientiel touristique fait écho à la stimulation expérientielle présentée par Christine Petr dans son article de 2014. Celle-ci propose une forme d'accompagnement du voyageur pour les professionnels du tourisme, par le biais d'un triptyque d'alternatives: "Aider à vivre, permettre le métarécit de l'expérience, faire visualiser le vécu» (Petr, 2014, p. 41) sont autant de moyens qui, accompagnés 
d'éléments contributeurs, vont faciliter la construction de l'expérience touristique. Ces derniers sont constitués des éléments de mise en scène de l'expérience visant à aider le touriste à ressentir son expérience : l'accompagnement par des guides, le décor du lieu et les plateformes d'aide à l'hébergement notamment (Ibid.). À ces dispositions d'ordre organisationnel s'ajoutent les moyens permettant au touriste de raconter son expérience (réseaux sociaux, blogs) en vue de nourrir son interaction à l'expérience, en lui permettant de la présenter à autrui. Ce qui renvoie à la possibilité pour le touriste de bénéficier d'outils lui permettant de prendre conscience de son vécu expérientiel au moment même où il le vit : les « témoignages du jour [...], l'affichage numérique des visages des participants à une attraction dans un parc de loisir [...] permettent en cela de visualiser et de cristalliser les ressentis » (Ibid., p. 41).

De la même manière, le positionnement expérientiel mentionné par l'auteur consiste à identifier et différencier les offres touristiques en fonction de leurs caractéristiques : hyper-réalité (parc à thème avec un univers factice), authenticité (volonté du touriste de sortir des sentiers touristiques, fabriqués pour vivre une expérience nourrie par des échanges en direct avec les habitants locaux, par exemple en vivant chez eux, etc.) ou altérité (désir de s'immerger dans la population locale au point de la ressentir).

Si les retombées positives ne sont pas toujours visibles pour les acteurs économiques et institutionnels (Camus, 2014), l'expérience constitue néanmoins une option pertinente pour le tourisme, dans la mesure où elle vise à reconnecter l'individu à ce qui a pour lui du sens. À cet égard, «l'analyse des dimensions subjectives, symboliques et émotionnelles liées à l'expérience au cours d'un processus d'achat ou de consommation afin de pouvoir les intégrer dans des stratégies permettant la création d'un avantage concurrentiel " (Batat et Frochot, 2014, p. VII) doit constituer un objectif pour les marketeurs et autres acteurs du tourisme.

La combinaison de différents éléments de contexte expérientiel représente un levier d'action pour ces derniers : ils peuvent, d'une part, positionner leur offre sur le ressenti émotionnel que celle-ci est susceptible de provoquer chez le touriste ; et, d'autre part, concevoir la mise en scène, l'intrigue et l'action (Filser, 2002) autour de leur prestation touristique, en prévoyant la participation du touriste.

La cohérence de l'initiative constitue dès lors une démarche stimulante pour tout acteur du tourisme, désireux de participer à l'élaboration d'un nouveau rapport à l'expérience: celui d'une synergie favorisant les conséquences positives inattendues qui peuvent résulter pour le touriste dans son désir de réenchanter ses actes de consommation (Ritzer, 2010). 


\section{BIBLIOGRAPHIE}

R. AMIROU, Imaginaire du tourisme culturel, Plon, 2000.

E. ARNOULD, L. PRICE et G. ZINKHAN, Consumers, McGraw-Hill/Irwin, 2002.

E. ARNOULD et C. THOMPSON, « Consumer culture theory (CCT): Twenty years of research ", Journal of Consumer Research, $\mathrm{n}^{\circ}$ 31(4), 2005, p. 868-882.

W. BATAT et I. FROCHOT, Marketing expérientiel. Comment concevoir et stimuler l'expérience client, Dunod, 2014.

C. BERTHO-LAVENIR, La roue et le stylo. Comment nous sommes devenus touristes, Odile Jacob, 1999.

A. BHATTACHARJEE et C. MOGILNER, « Happiness from ordinary and extraordinary experiences », Journal of Consumer Research, $\mathrm{n}^{\circ}$ 41(1), 2014, p. 1-17.

O. BOoRSTIN, The Image: A Guide to Pseudo-Events in America, New York : Harper and Row, 1964.

D. BOUDER-PAILLER et L. DAMAK, « Le design de l'espace de représentation d'un spectacle vivant influence-t-il les attitudes et comportements des spectateurs? Approche exploratoire », Actes du colloque sur le design, 2004.

D. BOURGEON et M. FILSER, « Les apports du modèle de recherches d'expériences à l'analyse du comportement dans le domaine culturel. Une exploration conceptuelle et méthodologique ", Recherche et applications en marketing, $\mathrm{n}^{\circ}$ 10(4), 1995, p. 5-25.

D. BOURGEON-RENAULT et P. BOUCHET, « Marketing expérientiel et analyse des logiques de consommation du spectacle sportif », Revue française du marketing, $n^{\circ} 21(2), 2007$.

D. BOURGEON-RENAULT, C. URBAin, A. GOMBAULT, M. LE GALL-ELY et C. PETR, « Gratuité et valeur attachée à l'objet de consommation culturelle : le cas des musées et des monuments ", Décisions marketing, 2009, p. 51-62.

S. CAMUS, « Marketing expérientiel, production d'expérience et expérience de consommation », Revue-Espaces, $\mathrm{n}^{\circ}$ 320, 2014, p. 28-37.

L. CARBone, Clued In: How to Keep Customers Coming Back Again and Again, Prentice-Hall, Upper Saddle River, NJ, 2004.

A. CARÙ et B. COVA, « Retour sur le concept d'expérience : pour une vue plus modeste et plus complète du concept », Actes des $7^{\text {èmes }}$ Journées de recherche en marketing de Bourgogne, 2002, p. 154-172.

A. CARÙ et B. COVA, « Approche empirique de l'immersion dans l'expérience de consommation : les opérations d'appropriation », Recherche et applications en marketing, $\mathrm{n}^{\circ}$ 18(2), 2003.

A. CARÙ et B. COVA, « Expériences de marque : comment favoriser l'immersion du consommateur?", Décisions marketing, 2006, p. 43-52.

A. CARÙ et B. cova, Consuming experience, Routledge, 2007.

M. DE CERTEAU, L'invention du quotidien. I. Arts de faire, Gallimard, 1990.

M. DE CERTEAU, L. Giard et P. Mayol, Les Arts de faire, l'invention du quotidien. II. Habiter, cuisiner, Gallimard, 1994. 
V. CHESNEAUX, « Une socio-anthropologue dans les coulisses de Disneyland Paris », Socioanthropologie, $\mathrm{n}^{\circ}$ 2, 1997 [https://socio-anthropologie.revues.org/36].

COFREMCA, Cofremca. Rapport, 1990 [http://www.sociovision.com].

E. COHEN, « Rethinking the Sociology of Tourism », Annals of Tourism Research, n 1, 1979,

p. $164-182$.

E. COHEN, « Authenticity and Commoditization in Tourism », Annals of Tourism Research, $\mathrm{n}^{\circ}$ 15(2), 1988, p. 371-386

L. COURNARIE, L'expérience, Philopsis, 2010 [http://philopsis.fr/IMG/pdf_ExperienceCournarie.pdf].

M. DUPUIS et D.L. SAVREUX, « Marketing expérientiel et performances des enseignes de distribution », Revue française du marketing, $\mathrm{n}^{\circ}$ 198.3(5), 2004.

ÉQUIPE MIT, Tourismes 1 : lieux communs, Belin, vol. 1, 2008.

M. FILSER, « Le marketing de la production d'expérience : statut théorique et implications managériales », Décisions marketing, 2002, p. 13-22.

M. FILSER, "Vingt ans de recherches en comportement du consumer », Sociétés, consommation et consommateurs, 2003, p. 15-20.

M. FILSER, « Préface », dans Le marketing sensoriel. De la stratégie à la mise en œuvre, Vuibert, 2007.

M. FILSER, « L'expérience de consommation : concepts, modèles et enjeux managériaux », Recherche et applications en marketing, $\mathrm{n}^{\circ}$ 23(3), 2008, p. 1-4.

A. FIRAT, N. DHOLAKIA et A. VENKATESH, « Marketing in Postmodern World », European Journal of Marketing, n² 29(1), 1995, p. 40-56.

S. FLAMBART-RUAUD, « Les évolutions du concept de marketing », Décisions marketing, 1997, p. 7-20.

G. FLAUBERT, Voyage en Orient (1849-1851), Gallimard, 2006.

I. FROCHOT et W. BATAT, Marketing and Designing the Tourist Experience, Oxford : Goodfellow Publishers Limited, 2013.

W. FUX, « Designer une expérience touristique en dix points clés », Revue-Espaces, n 321, 2014.

E. GOFFMAN, The Presentation of Self in Everyday Life, Harmondsworth : Penguin, 1959.

N. GRABURN, Ethnic and tourist arts: Cultural expressions from the fourth world, California : Universisty of California Press, 1976.

N. GRABURN, « The anthropology of tourism », Annals of Tourism Research, n 10, 1983, p. 9-33.

N. GRABURN, « Tourism: the sacred journey », See Smith, 1989, p. 9-33.

C. GRÖNROOS, « Quo Vadis, Marketing Toward Relationship Marketing », Journal of Marketing Management, $\mathrm{n}^{\circ}$ 5, 1994, p. 347-360.

P. HETZEL, Planète conso : marketing expérientiel et nouveaux univers de consommation, Paris : Éditions d'Organisation, 2002.

M. HOLBRoOK, « The millennial consumer in the texts of our times: Experience and entertainment », Journal of Macromarketing, $n^{\circ}$ 20(2), 2000, p. 178-192.

M. HOLBROOK et E. HIRSCHMAN, « The Experiential Aspects of Consumption: Consumer Fantasies, Feelings and Fun ", Journal of Consumer Research, n 9(2), 1982, p. 12-140. 
J. JAFARI et J.B. RITCHIE, « Toward a framework for tourism education: Problems and prospects », Annals of Tourism Research, $\mathrm{n}^{\circ}$ 8(1), 1981, p. 13-34.

J. JAFARI, « Le système du tourisme : modèles socio-culturels en vue d'applications théoriques et pratiques ", Society and Leisure, $\mathrm{n}^{\circ} 11(1), 1988$.

R. KNAFOU, L'Institut de Saint-Gervais : une recherche-action dans la montagne touristique, Belin, 1997.

P. KOTLER, « Atmospherics as a marketing tool », Journal of Retailing, n 49(4), 1973, p. 48-64.

R. KOZINET, J. SHERRY, B. DEBERRY-SPENCE, A. DUHACHEK, K. NUTTAVUTHISI et al., « Themed flagship brand stores in the new millenium: theory, practice, prospects ", Journal of Retailing, $\mathrm{n}^{\circ} 78,2002$, p. 17-29.

R. LADWEIN, « Voyage à Tikidad : de l'accès de l'expérience de consommation », Décisions marketing, $\mathrm{n}^{\circ} 28,2002$, p. 53-63.

R. LAING, La politique de l'expérience, Stock, 1969.

D. LASALLE et T. BRITTON, Priceless: Turning ordinary products into extraordinary experiences, Harvard Business Press, 2003.

A. LEBRUN, Les expériences recherchées au cour des attentes des touristes, La lettre de l'OCIM, 2005.

G. LIPOVETSKI, Le Bonheur paradoxal, Paris : Gallimard, 2006.

Y. LOCHARD, « L'avènement des "savoirs expérientiels" », La Revue de l'Ires, n 3(55), 2007, p. 79-95.

D. MACCANNELL, « Staged Authenticity: Arrangements of Social Space in Tourist Settings ", American Journal of Sociology, $\mathrm{n}^{\circ}$ 79(3), 1973, p. 589-603.

M. MAFFESOLI, Au creux des apparences : pour une éthique de l'esthétique, Plon, 1990.

G. MARION, « Le marketing relationnel existe-t-il ? », Décisions marketing, 2001, p. 7-16.

S. MATSON-BARKAT, Des expériences sensorielles aux sens des expériences : une approche CCT de l'expérience des touristes au restaurant, Rennes : Université de Rennes 1, 2012.

R. MENCARELLI, « Conceptualisation et mesure de la valeur perçue d'un lieu de consommation. Application au domaine du spectacle vivant ", $21^{\text {ème }}$ Colloque de l'Association française de marketing, 2005.

R. MENCARELLI et S. MARTEAUX, « Positionnement stratégique des entreprises culturelles : proposition d'enrichissement autour du concept de valeur », Management \& avenir, $n^{\circ} 3(5), 2005$, p. 161-178.

J. MICHAUD, « Anthropologie, tourisme et sociétés locales au fil des textes », Anthropologie et sociétés, $\mathrm{n}^{\circ}$ 25(2), 2001.

C. PETR, « Le tourisme expérientiel, ça n'existe pas... mais c'est utile à la réflexion marketing ", Revues- Espaces, 2014.

B. PINE et J. GILMORE, « Welcome to the experience economy », Harvard Business Review, $\mathrm{n}^{\circ} 76,1998$, p. 97-105.

B. PINE et J. GILMORE, The experience economy: work is theatre \& every business a stage, Harvard Business Press, 1999.

C. PRAHALAD et V. RAMASWAMY, « Co-creation experiences: the next practice in value creation », Journal of Interactive Marketing, $\mathrm{n}^{\circ}$ 18(3), 2004, p. 5-14. 
M. PULH et R. MENCARELLI, « Muséo-parcs et réenchantement de l'expérience muséale. Le cas de la Cité des arts et des sciences de Valencia », Décisions marketing, $n^{\circ}$ 60, 2010, p. 21-31.

G. PUNJ, N. GIRISH, W. DAVID et D. STEWART, « An interaction framework of consumer decision making ", Journal of Consumer Research, 1983, p. 181-196.

D. RATTI, Entre pouvoir et résistance : la place de l'individu dans les sociétés technocratiques, Montréal, Canada : Université Concordia, 2002 [http://spectrum.library.concordia.ca/1884/1/ MQ72876.pdf].

J. RIFKIN, La nouvelle société du coût marginal zéro, Actes Sud, Babel essai, 2016.

S. RIEUNIER, « Le marketing sensoriel chez Nature et Découvertes », Décisions marketing, n 33, 2004.

G. RITZER, Enchanting a disenchanted world: continuity and change in the cathedrals of consumption, Pine Forge Press, 2010.

C. ROEDERER, Marketing et consommation expérientiels, EMS, 2012.

B. schмiтt, Experiential Marketing. How to Get Customers To Sense, Feel, Think, Act and Relate to Your Company and Brands, New York: The Free Press, 1999.

B. SCHMITT, Customer Experience Management: A Revolutionary Approach to Connecting with Your Customers, New York : Wiley, 2003.

M.J. SHEN, « The effects of globalized authenticity on souvenir », International Journal of Innovative Management, Information \& Production, $\mathrm{n}^{\circ}$ 2(1), 2011, p. 68-76.

A. STRONZA, « Anthropology of tourism: Forging new ground for ecotourism and other alternatives ", Annual Review of Anthropology, 2001, p. 261-283.

V. TURNER, The Ritual Process: Structure and Anti-structure, Chicago : Aldine, 1969.

V. TURNER, From Ritual to Theater: The Human Seriousness of Play, New York : PAJ, 1982.

V. TURNER et E. TURNER, Image and Pilgrimage in Christian Culture: Anthropological Perspective, Columbia : Columbia University Press, 1978.

J. TRINQUECOSTE, « Pour une clarification théorique du lien marketing-stratégie », Recherche et applications en marketing, $\mathrm{n}^{\circ}$ 14(1), 1999, p. 59-80.

S. VARGO et R. LUSCH, « Service-dominant logic: continuing the evolution », Journal of the Academy of Marketing Science, $\mathrm{n}^{\circ}$ 36(1), 2008, p. 1-10.

M. WOLF, The entertainment economy. The mega-media forces that are re-shaping our lives, New York : Penguin Books, 1999.

\section{NOTES}

1. Dans la littérature, l'origine de la pratique du tourisme est généralement située au XIX ${ }^{\mathrm{e}}$ siècle, avec la création de voyages organisés par Thomas Cook (dès 1840). Son activité se développe notamment grâce à l'Exposition universelle de Londres, en 1851.

2. Notre traduction : l'auteur entend par «back » l'endroit où les membres du personnel peuvent se retirer pour se relaxer et se préparer.

3. Constituée de pas moins de 14 enseignants-chercheurs en géographie et spécialistes du tourisme, l'équipe MIT (Mobilités, itinéraires, tourisme) créée en 1994 sous l'impulsion de Rémy Knafou - professeur émérite de l'Université Paris 1 Panthéon-Sorbonne - a consacré une partie 
de ses recherches à la mise en tourisme des lieux et à la mise en valeur des mobilités. Le laboratoire de recherche a notamment produit une série de trois ouvrages, initiée en 2002 par Tourismes 1, Lieux communs (Paris, Belin, Coll. Mappemonde), suivie en 2005 par Tourismes 2, Moments de lieux (Ibid.) et en 2011 par Tourismes 3 : la révolution durable (Ibid.).

4. Cofremca, créé en 1954 et devenu Sociovision en 1998, est un organisme privé qui accompagne les clients privés - entreprises françaises du CAC 40 et autres grandes entreprises internationales ou autres structures paritaires ou institutionnelles - dans la compréhension de l'évolution de la société française en vue de mieux appréhender ses changements pour une meilleure réaction et adaptation. L'organisme dispose, pour ce faire, d'un large éventail d'outils méthodologiques qualitatifs et quantitatifs - pour mener les études et rapports.

5. Notre traduction.

6. Voir : http://paris.danslenoir.com/fr/frontpage/ (consulté le 15 septembre 2016)

7. Voir : http://www.puydufou.com/fr/ (consulté le 15 septembre 2016)

8. Voir : http://www.natureetdecouvertes.com/notre-histoire (consulté le 15 septembre 2016)

9. Voir : http://www.fontevraud.fr/ (consulté le 15 septembre 2016)

10. L'Abbaye de Fontevraud désigne les touristes en leur qualité d'hôtes et non de visiteurs, en référence aux valeurs d'hospitalité définies par le fondateur de l'Abbaye en 1110, Robert d'Arbrissel.

11. Voir : http://www.angers.fr/actualites/detail/article/12162-le-quai-version-2015-2016-leprogramme-est-sorti/ (consulté le 15 septembre 2016).

12. Extrait d'un entretien d'1h19 le 7 juillet 2016 avec le chef de rang de l'Abbaye Royale de Fontevraud.

\section{RÉSUMÉS}

L'article propose une revue de la littérature multidisciplinaire du concept d'expérience et de son opérationnalisation. Il rappelle les prémices et enjeux d'une approche expérientielle qui, en particulier en marketing, a connu des évolutions en termes de conceptualisation et de modélisation, permettant de mieux comprendre et appréhender la consommation, notamment dans le secteur du tourisme. Après la synthèse de la littérature, sont développés et illustrés les outils et processus de la mise en œuvre d'une démarche expérientielle. Ces derniers reposent sur deux principes fondamentaux : la participation du touriste, ainsi que la mise en scène du lieu et de l'offre touristique.

The article proposes a multidisciplinary literature review of the concept of experience and its operationalization. It reminds the beginnings and stakes of an experiential approach which, in particular in marketing, knew evolutions in terms of conceptualization and modelling, allowing to better understand consumption, especially in the sector of tourism. After the synthesis of the literature, are developed and illustrated tools and process of the implementation of an experiential approach. These latter are based on two fundamental principles: the participation of the tourist, as well as the stage of the place and of the tourist offer. 


\section{AUTEURS}

\section{SOLÈNE BARGAIN}

Doctorante en sciences de gestion, Université d'Angers, GRANEM

SANDRA CAMUS

Professeur des universités en sciences de gestion, Université d'Angers, GRANEM, Esthua

Tourisme et Culture 